\title{
An Extended Family with Familial Medullary Thyroid Carcinoma and Hirschsprung's Disease
}

\author{
Takehito Igarashi ${ }^{1}$, Ritsuko Okamura ${ }^{1}$, Tomoo Jikuzono ${ }^{1}$, \\ Shinya Uchino ${ }^{2}$, Iwao Sugitani ${ }^{1}$ and Kazuo Shimizu ${ }^{1}$ \\ ${ }^{1}$ Division of Endocrine Surgery, Department of Surgery, Nippon Medical School \\ ${ }^{2}$ Department of Surgery, Noguchi Hospital
}

\begin{abstract}
Familial medullary thyroid carcinoma (FMTC) is an autosomal dominant inherited disease that has highly characteristic clinical features, including medullary thyroid carcinoma (MTC). Mutation of the RET proto-oncogene is known to be responsible for development of FMTC and for multiple endocrine neoplasia types $2 \mathrm{~A}$ and $2 \mathrm{~B}$. Hirschsprung's disease is the most common form of structural intestinal obstructive disease in human newborns. Hirschsprung's disease is defined by the absence of neural crest-derived enteric ganglia along a variable length of the bowel that invariably involves the rectoanal junction. Co-segregation of FMTC and Hirschsprung's disease is uncommon; nevertheless, in 3 generations of 1 family, we observed 5 patients with FMTC, 2 patients with Hirschsprung's disease, and 1 patient with characteristics of both FMTC and Hirschsprung's disease. Moreover, a Cys620Ser mutation in RET was identified in 4 of the 8 patients. This mutation had both activating and inactivating effects on the RET (REarranged during Transfection) protein. There were individual differences in the penetrance of Hirschsprung's disease due to the RET mutation, but the penetrance of MTC was uniform and high. Genetic testing is important for making decisions about treatment and follow-up in families of this kind.

(J Nippon Med Sch 2014; 81: 64-69)
\end{abstract}

Key words: familial medullary thyroid carcinoma, Hirschsprung's disease, RET

\section{Introduction}

Hereditary medullary thyroid carcinoma (MTC) is autosomal dominant inherited disease and occurs in multiple endocrine neoplasia type 2A (MEN2A) and type 2B (MEN2B), as well as in familial MTC (FMTC). Each of these diseases has been associated with activating mutations of the RET proto- oncogene ${ }^{1.2}$. Several RET mutations cause activation of the RET gene and facilitate cellular signal transduction $^{3.4}$.

The most common germ-line mutations involve the extracellular domain of RET at codons 609,611 , 618, 620, 630, and $634^{5-8}$. Mutations occur in the tyrosine kinase domain of RET at codons 768,790 , 791,804 , and $891^{5-8}$.

Hirschsprung's disease is the most common type

Correspondence to Takehito Igarashi, MD, PhD, Division of Endocrine Surgery, Department of Surgery, Nippon Medical School, 1-1-5 Sendagi, Bunkyo-ku, Tokyo 113-8603, Japan

E-mail: takehito@nms.ac.jp

Journal Website (http://www.nms.ac.jp/jnms/) 


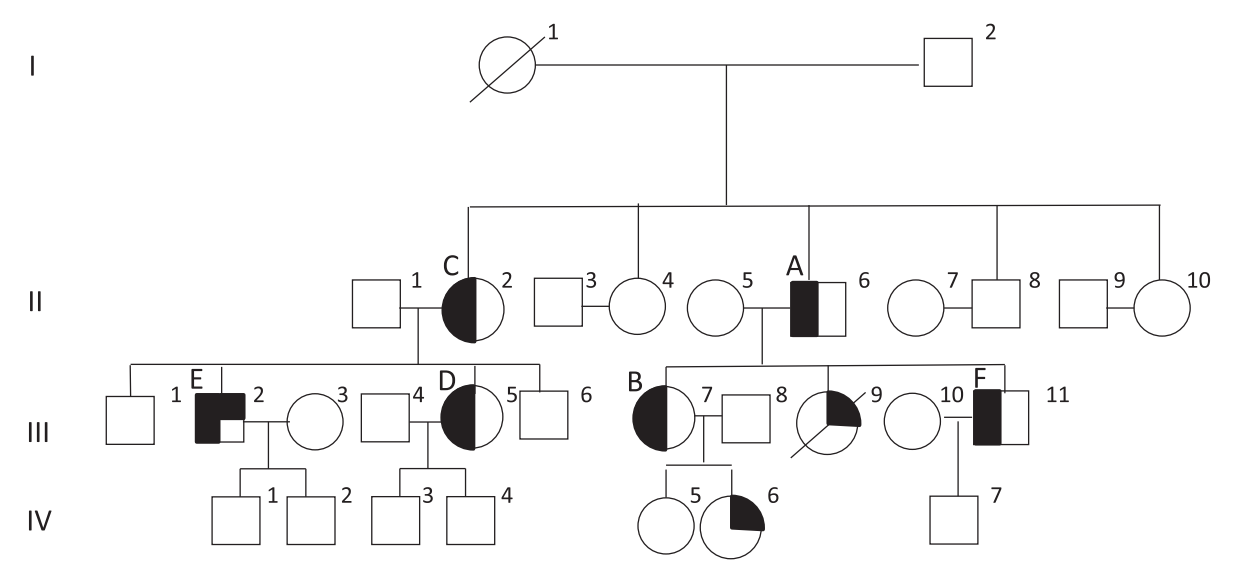

Hirschsprung's disease
Medullary thyroid carcinoma
Hirschsprung's disease and medullary thyroid carcinoma

Fig. 1 The pedigree of the described family.

Three patients presented with MTC, 3 patients presented with Hirschsprung's disease, and 1 presented with concomitant MTC and Hirschsprung's disease; each patient is indicated in this pedigree. Two patients presented with Hirschsprung's disease but did not undergo genetic testing.

of structural intestinal obstructive disease in human newborns. It is characterized by the absence of neural crest-derived enteric ganglia along a variable length of the bowel that invariably includes the rectoanal junction. Hirschsprung's disease is associated with various chromosomal abnormalities and inherited disorders. The major susceptibility gene is RET, and RET mutations have been identified in $50 \%$ of familial cases and $15 \%$ to $35 \%$ of sporadic cases of Hirschsprung's disease ${ }^{12}$.

The RET proto-oncogene, which is critical to the development of MTC and of Hirschsprung's disease, encodes RET (REarranged during Transfection), a transmembrane receptor-type kinase that is expressed in cells derived from the neural crest and the urogenital tract.

A single RET mutation rarely causes both activation and inactivation of RET signaling. Reportedly, the RET gene is deeply involved in the development of MEN2, FMTC, and Hirschsprung's disease $^{5-7}$. The clinical severities of Hirschsprung's disease due to different RET mutations are highly individual and, as a result, show incomplete penetrance (-50\%). Therefore, only affected persons in whom MTC develops are recognized in family pedigrees of these diseases. We examined data from an extended family in which Hirschsprung's disease, MTC, and 1 case of concomitant Hirschsprung's disease and MTC were evident; notably, a codon 620 RET mutation was segregating in this family.

\section{Materials and Methods}

This study was retrospective, and each patient gave written informed consent to participate. Data were extracted from medical records; additional medical interviews and laboratory examinations were also conducted. Genetic tests were performed following an independent review by the Institutional Independent Review Board and according to national guidelines. Subjects were free to withdraw from the study at any time. Interviews about the presence of the diseases in the family were conducted, and a pedigree was created.

\section{Summary of Patients (Fig. 1)}

Patient A (II-6): A 57-year-old man presented with neck swelling during a medical check-up and asked us to investigate it. Close inspection resulted in the diagnosis of MTC. The family history revealed a 
T. Igarashi, et al

Table 1 Overview of preoperative data

\begin{tabular}{lccccc}
\hline Patient & $\begin{array}{c}\text { Age at operation } \\
\text { (years) }\end{array}$ & Sex & $\begin{array}{c}\text { Calcitonin } \\
(\mathrm{pg} / \mathrm{mL})\end{array}$ & $\begin{array}{c}\text { Carcinoembryonic } \\
\text { antigen (ng/mL) }\end{array}$ & RET mutation \\
\hline A (II-6) & 57 & $\mathrm{M}$ & 670 & 8.5 & 620 TGC to TCC \\
B (III-7) & 33 & $\mathrm{~F}$ & 32 & 0.6 & 620 TGC to TCC \\
C (II-2) & 64 & $\mathrm{~F}$ & 390 & 10.7 & Testing not done \\
D (III-5) & 33 & $\mathrm{~F}$ & 340 & 23.6 & Testing not done \\
E (III-2) & 40 & $\mathrm{M}$ & 733 & 5.1 & 620 TGC to TCC \\
F (III-11) & 33 & $\mathrm{M}$ & 1,134 & 6.2 & 620 TGC to TCC \\
\hline
\end{tabular}

daughter (III-9) who had died of Hirschsprung's disease as an infant, and a grandchild who had been treated for Hirschsprung's disease. Additionally, his older sister had undergone right lobectomy of the thyroid because of MTC. Therefore, we concluded that the MTC in patient A was not sporadic but familial. We recommend genetic testing, and the patient agreed. His siblings were screened (the value of carcinoembryonic antigen [CEA] and calcitonin were determined, and the thyroid glands were evaluated with ultrasonography) at the same time, and no abnormalities were found other than a mass in the residual thyroid gland in an older sister.

Patient B (III-7): A 33-year-old woman whose daughter (IV-6) had been treated for Hirschsprung's disease agreed to undergo genetic testing. She had no thyroid symptoms, but she wanted to undergo prophylactic total thyroidectomy because of an RET mutation.

Patient C (II-2): A 64-year-old woman was found to have recurrent MTC in the residual thyroid gland; she decided to undergo complementary total thyroidectomy but refused genetic testing.

Patient D (III-5): A 33-year-old woman was worried about increased levels of CEA after surgery for breast cancer and visited our hospital. She was found to have MTC but refused genetic testing.

Patient E (III-2): A 40-year-old man, whose mother and young sister had undergone operations for MTC, had undergone 4 different colorectal operations as a child because of Hirschsprung's disease; he consulted another hospital, where MTC was diagnosed, and agreed to undergo genetic testing.

Patient F (III-11): A 33-year-old man wanted to have the thyroid checked because his father and sister had undergone surgery for MTC. After close inspection, he was also found to have MTC and agreed to undergo genetic testing.

Preoperative values of CEA and calcitonin for each of these patients are shown in Table $\mathbf{1}$.

\section{Gene Analysis}

A DNA extractor WB kit (Wako Pure Chemical Industries, Ltd., Osaka, Japan) was used according to the manufacturer's instructions to extract genomic DNA from samples of peripheral blood. The absorbance at $260 \mathrm{~nm}$ was determined with a spectrophotometer (SmartSpec 3000, Bio-Rad Laboratories, Inc., Hercules, CA, USA) to measure the DNA concentration in each sample of genomic DNA.

Exons 10, 11, and 13-16 of RET were amplified from genomic DNA from patient A via the polymerase chain reaction (PCR); previously published primers and PCR conditions were used. ${ }^{8}$. A mutation was discovered in exon 10 in genomic DNA from patient $\mathrm{A}$, and all subsequent analyses of RET in DNA samples from this family were restricted to exon 10.

Amplified PCR fragments were purified with a purification kit (QIAquick PCR Purification Kit, Qiagen, Hilden, Germany) and subjected to additional PCR with sense or antisense primers and reaction mix (ABI PRISM Big Dye Terminator v1.1 Cycle Sequencing Ready Reaction mix, Applied Biosystems, Foster City, CA, USA). Each sample containing resultant $\mathrm{PCR}$ products was eluted through a spin column (Centri-Sep, Applied Biosystems), and the PCR products were sequenced on an automated capillary DNA sequencer (ABI PRISM 3100, Applied Biosystems). 
A Kindred with FMTC and Hirschsprung's Disease

Table 2 Overview of operative findings

\begin{tabular}{llcl}
\hline Patient & \multicolumn{1}{c}{ Operation } & Time $(\mathrm{min})$ & Postoperative diagnosis \\
\hline A (II-6) & Total thyroidectomy+D3a & 168 & pT1(m), pN1b, pEx0 \\
B (III-7) & Total thyroidectomy+CND & 94 & pT1(m), pN0, pEx0 \\
C (II-2) & Total thyroidectomy+D3a & 65 & pT2(m), pN0, pEx0 \\
D (III-5) & Total thyroidectomy+D3a & 230 & pT1(m), pN0, pEx0 \\
E (III-2) & Total thyroidectomy+CND & 107 & pT1, pN1a, pEx0 \\
F (III-11) & Total thyroidectomy+CND & 70 & pT1(m), pN0, pEx0 \\
\hline
\end{tabular}

D3a, bilateral lateral lymph node dissection; CND, central lymph node dissection

\section{Results}

In this extended family, 6 persons were found to have MTC and underwent surgery. Four of the 6 were found to carry a Cys620Ser mutation of RET. Each surgery was performed without incident and resulted in a successful outcome (Table 2). None of the patients had recurrent laryngeal nerve palsy or hypoparathyroidism. The recovery period for patient $\mathrm{F}$ has been too short to reliably evaluate the outcome, as he underwent the operation only 2 months ago as of this writing. More than 5 years have passed since surgery for the other patients with MTC, and none have shown any evidence of recurrence; however, the CEA and calcitonin levels in patient $\mathrm{C}$ remain high.

Three patients with Hirschsprung's disease were identified, but 2 of them did not undergo genetic testing (Fig. 1, Table 1). One of these patients presented with concomitant FMTC and Hirschsprung's disease and carried the Cys620Ser mutation of RET.

It is extremely important to carefully monitor the course of each patient over the long term. Four of the 6 patients in the present family were found to have an RET mutation that could account for the disease. In each of these patients DNA sequencing revealed a Cys620Ser mutation of the RET gene (Table 1).

Because we did not obtain parental consent, we did not perform genetics tests of samples from a daughter of patient B. However, the daughter has never had a mass of the thyroid gland or elevated serum levels of calcitonin or CEA. Our analysis of this family suggests that patient B's daughter should also be followed up carefully because, on the basis on the family history, MTC is expected to develop.

\section{Discussion}

Co-segregation of FMTC and Hirschsprung's disease is uncommon, but in 1 extended family we observed 5 patients with FMTC, 3 patients with Hirschsprung's disease, and 1 patient who showed characteristics of both FMTC and Hirschsprung's disease. Co-segregation of FMTC and Hirschsprung's disease relates mostly to the cysteine-rich area at codon 620 of RET. All of the patients were related to one another, and 4 of them were found to carry the same RET allele, Cys620Ser.

Codon 620 of RET has been called the "Janus gene" because, like the Roman god of doorways, it can face in both directions; RET gain-of-function mutation causes MTC, and RET loss-of-function mutation causes Hirschsprung's disease.

RET is important for cellular growth and differentiation and is involved in several types of multiple neoplasia, such as MEN2A, MEN2B, and FMTC $^{1,2}$. Activation patterns of a number of RET mutations have been reported to be associated with certain types of disease ${ }^{3.4}$. Several large-scale studies have demonstrated a correlation between RET mutations and disease phenotypes ${ }^{14}$. The Cys634Arg mutation results in MEN2A, and Met918Thr results in self-dimerization and constitutive RET activation, leading to abnormal cell growth and dysfunction of differentiation ${ }^{5-7}$. These findings indicate that RET activation is responsible for the pathogenesis of these diseases.

Hirschsprung's disease is one of the most common diseases caused by malformation of neural crest- 
derived enteric structures in neonates led by dysfunction of RET. Butter et al. have reported on 20 patients with Hirschsprung's disease who each carried a mutation of RET; each patient also underwent prophylactic thyroidectomy and was ultimately healthy and cancer-free ${ }^{9}$. Hirschsprung's disease is rarely reported with other neoplastic diseases. Patients with Hirschsprung's disease and MTC have been reported in a single family ${ }^{10}$; however, co-segregation of FMTC and Hirschsprung's disease is rare.

Here, 4 persons with a mutation in codon 620 of RET gene were found in a single family, but how this mutation could result in the diseases observed in each individual is difficult to understand.

The biological effects of codon 620 of RET have been studied to understand RET gain-of-function mutations and the development of MTC and to understand RET loss-of-function mutations and the development of Hirschsprung's disease.

Several studies have demonstrated that the cysteine mutations in the extracellular domain activate RET by inducing covalent RET dimerization $^{1,3}$. This molecular mechanism could account for RET gain-of-function mutations ${ }^{11,13}$.

Codon 620 RET mutation decreases expression of the cell-surface form of RET protein. These mutations appear to impair the transport of RET to the plasma membrane or the proper maturation of $\mathrm{RET}^{13}$.

The RET protein and glial cell line-derived neurotrophic factor family (GDNF) receptor $\alpha 1$ (GFR $\alpha 1$ ) form a signaling receptor complex for GDNF. In particular, signaling by GDNF promotes the survival of dopaminergic neurons. Arighi et al. ${ }^{11}$ have demonstrated that cells carrying a mutation in codon 620 of RET are unable to migrate and branch in response to GDNF. Moreover, insensitivity to GDNF renders cells more prone to apoptosis. These features are considered to be loss-of-function phenotypes $^{11}$.

Whole-gene analyses of RET, gene-expression profiling, and whole-genome sequencing can be useful for revealing the disease mechanisms and the signal transduction pathway of RET. The results of the present study suggest that sequencing of RET should be performed in cases of FMTC or Hirschsprung's disease and in high-risk carriers with a hereditary background as a clinical guideline for FMTC, Hirschsprung's disease, and MEN2 ${ }^{14}$.

Conflict of Interest: None of the authors have any potential conflicts of interest associated with this research.

\section{References}

1. Hofsttra RM, Lansvater RM, Ceccheri I, Stlup RP, Stelwagen T, et al: A mutation in the RET protooncogene associated with multiple endocrine type $2 \mathrm{~B}$ and sporadic medullary thyroid carcinoma. Nature 1994; 367: 375-376.

2. Raue F, Frank-Raue K: Genotype-phenotype relationship in multiple endocrine neoplasia type 2 Implication for clinical management. Horomones (Athens) 2009; 8: 23-28.

3. Santoro M, Carlomagno F, Romano A, Bottaro DP, Dathan NA, et al.: Activation RET as a dominant transforming gene by germ line mutations of MEN2A and MEN2B. Science 1995; 267: 381-383.

4. Berndt I, Reuter M, Saller B, et al:. A new hot spot for mutation the ret proto-oncogene causing familial medullary thyroid carcinoma and multiple endocrine neoplasia type 2A. J Clin Endocrinol Metab 1998; 83: 770-774.

5. Mulligan LM, Eng C, Healy CS, Clayton D, Kwok JB, et al: Specific mutations of RET proto-oncogene are related to disease phenotype in MEN 2A and FMTC. Nat Genet 1994; 6: 70-74.

6. Romei C, Elisei R, Prinhera A, Coccherini I, Mancusi $\mathrm{F}$, et al: Somatic mutations of the ret proto-oncogene in sporadic medullary thyroid carcinoma are not restricted to exon 16 and are associated with tumor recurrence. J Clin Endocrinol Metab 1996; 81: 16191622.

7. Schuffenecker I, Virally-Mond M, Brohet R, Goldgar D, Conte-Devol B, et al.: Risk and penetrance of primary hyperparathyroidism in multiple endocrine neoplasia type $2 \mathrm{~A}$ families with mutations at codon 364 of the RET proto-oncogene; Groupe D'etude des Tumeurs aCalcitonine. J Clin Endocrinol Metab 1998; 83: 487-491.

8. Uchino S, Noguchi S, Adachi M, Sato M, Yamashita $\mathrm{H}$, et al: Novel point mutations and allele loss at the RET locus in sporadic medullary thyroid carcinomas. Jpn J Cancer Res 1998; 89: 411-418.

9. Butter A, Gangnel J, Al-Jazaeri A, Emran MA, Deal C, et al:: Prophylactic thydoidectomy in pediatric carries of multiple endocrine neoplasia type $2 \mathrm{~A}$ or familial medullary thyroid carcinoma: mutation in $\mathrm{C}$ 620 is associated with Hirschspung's disease. J Pediatr Surg 2007; 2: 203-206.

10. Fialkowski EA, DABenedetti MK, Moley JF, Bachrach B: RET proto-oncogene testing in infants presenting with Hirschsprung disease indentifies 2 new multiple neoplasia 2a in kidreds. J Pediatr Surg 
A Kindred with FMTC and Hirschsprung's Disease

2008; 43: 188-190.

11. Arighi E, Posueva A, Degl'innocenti D, Borrello MG, Carniti C, et al.: Biological effect of the dual phenotypic Janus mutation or ret segregating both multiple endocrine neoplasia type 2 and Hirschsprung's disease. Mol Endocrinol 2004; 18: 1004-1017.

12. Amiel J, Lyonnet S: Hirschsprung disease, associated syndromes, and genetics: a review. J Med Genet 2001; 38: 729-739.

13. Ito $\mathrm{S}$, Iwashita $\mathrm{T}$, Asai N, Murakami H, Iwata $\mathrm{Y}$, et al.: Biological Properties of Ret with Cysteine
Mutation Correlate with Mutiple Endocrine Neoplasia Type 2A, Familial Medullary Thyroid Carcinoma, and Hirschsprung's Disease Phenotype. CANCER RESEARCH 1997; 57: 2870-2872.

14. American Thyroid Association Guideline Task Force: Medullary thyroid cancer: management guidelines of the American Thyroid Association. Thyroid 2009; 19: 565-612.

(Received, June 14, 2013)

(Accepted, July 17, 2013) 